\title{
Precipitation and total power consumption in the ionosphere: Global MHD simulation results compared with Polar and SNOE observations
}

\author{
M. Palmroth ${ }^{1}$, P. Janhunen ${ }^{2,1}$, G. Germany ${ }^{3}$, D. Lummerzheim ${ }^{4}$, K. Liou ${ }^{5}$, D. N. Baker ${ }^{6}$, C. Barth ${ }^{6}$, \\ A. T. Weatherwax ${ }^{7}$, and J. Watermann ${ }^{8}$ \\ ${ }^{1}$ Space Research Division, Finnish Meteorological Institute, Helsinki, Finland \\ ${ }^{2}$ Department of Physical Sciences, University of Helsinki, Helsinki, Finland \\ ${ }^{3}$ University of Alabama in Huntsville, Huntsville, AL, USA \\ ${ }^{4}$ Geophysical Institute, University of Alaska, Fairbanks, AK, USA \\ ${ }^{5}$ Applied Physics Laboratory, The Johns Hopkins University, Laurel, MD, USA \\ ${ }^{6}$ Laboratory for Atmospheric and Space Physics, University of Colorado at Boulder, Boulder, CO, USA \\ ${ }^{7}$ Siena College, Department of Physics, Loudonville, NY, USA \\ ${ }^{8}$ Danish Meteorological Institute, Atmosphere Space Research Division, Farum, Denmark
}

Received: 22 July 2005 - Revised: 6 February 2006 - Accepted: 14 February 2006 - Published: 19 May 2006

\begin{abstract}
We compare the ionospheric electron precipitation morphology and power from a global MHD simulation (GUMICS-4) with direct measurements of auroral energy flux during a pair of substorms on 28-29 March 1998. The electron precipitation power is computed directly from global images of auroral light observed by the Polar satellite ultraviolet imager (UVI). Independent of the Polar UVI measurements, the electron precipitation energy is determined from SNOE satellite observations on the thermospheric nitric oxide (NO) density. We find that the GUMICS-4 simulation reproduces the spatial variation of the global aurora rather reliably in the sense that the onset of the substorm is shown in GUMICS-4 simulation as enhanced precipitation in the right location at the right time. The total integrated precipitation power in the GUMICS-4 simulation is in quantitative agreement with the observations during quiet times, i.e., before the two substorm intensifications. We find that during active times the GUMICS-4 integrated precipitation is a factor of 5 lower than the observations indicate. However, we also find factor of 2-3 differences in the precipitation power among the three different UVI processing methods tested here. The findings of this paper are used to complete an earlier objective, in which the total ionospheric power deposition in the simulation is forecasted from a mathematical expression, which is a function of solar wind density, velocity and magnetic field. We find that during this event, the correlation coefficient between the outcome of the forecasting expression and the simulation results is 0.83 . During the event, the
\end{abstract}

Correspondence to: $\mathrm{M}$. Palmroth

(minna.palmroth@fmi.fi) simulation result on the total ionospheric power deposition agrees with observations (correlation coefficient 0.8 ) and the $A E$ index (0.85).

Keywords. Ionosphere (Auroral ionosphere; Modeling and forecasting; Particle precipitation)

\section{Introduction}

The recently emerged need to predict the space environment requires a quantitative analysis of the global energy circulation in the solar wind-magnetosphere-ionosphere system. The first energy input-output analyses developed in the 1980's (e.g. Akasofu, 1981; Vasyliunas et al., 1982) determined qualitatively the most important factors in the solar wind governing the energy transfer and dissipation processes. However, a substantial amount of new information has become available as the space-borne measurement systems have become more sophisticated and the ground-based systems have been extended to world-wide networks. Consequently, more recent studies (e.g. Lu et al., 1998; Tanskanen et al., 2002) have shown that the quantitative picture of the energy transfer and dissipation is still not clear. For instance, in the 1980's it was thought that the ring current would be the largest energy sink within the magnetosphere, and that the ionosphere would only contribute to the details of the global energy budget (Akasofu, 1981). On the contrary, the current understanding is that from the total energy distributed in the ionosphere and the ring current, the ionosphere absorbs up to or over $50 \%$ both during storms (e.g. Lu et al., 1998; Baker et

Published by Copernicus GmbH on behalf of the European Geosciences Union. 
al., 2001; Pulkkinen et al., 2002) and substorms (Tanskanen et al., 2002).

Two main processes deposit solar wind energy in the ionosphere: Ohmic (Joule) heating caused by the ionospheric closure of field-aligned currents, and the thermal energy, which is left to the ionosphere as electrons from the magnetosphere precipitate to the ionosphere and collide with atmospheric particles. Of these, the Joule heating is thought to be the larger sink. Lu et al. (1998), for example, estimated that $\sim 20 \%$ of the total energy consumed by the ring current and the ionosphere would be due to precipitating electrons. The energy related to the electron precipitation affects the global energetics both directly and indirectly, since together with the solar EUV radiation the precipitation determines the ionospheric conductivity structure and therefore affects the global electric field (e.g. Kamide and Richmond, 1982). Hence the electron precipitation also affects the morphology and magnitude of global Joule heating. Thus, a thorough understanding of the structure and magnitude of the auroral energy flux is required in order to arrive at a quantitative estimate of the ionospheric total energy deposition.

As will be explained in Sect. 2, auroral light provides a diagnostic tool to study electron precipitation and the related energy flux. The auroral luminosity is produced as the atmospheric particles excited in collisions with the precipitating magnetospheric electrons return to the ground state releasing the extra energy as photons. Therefore, auroral brightness is related to the flux and energy of the precipitating electrons and can thereby be converted to auroral energy flux characteristics. This can be done either using groundbased all-sky cameras using visible light (Janhunen, 2001), or from space-borne global imagers using ultraviolet (UV) wavelengths (e.g. Lummerzheim et al., 1991; Germany et al., 1997; Liou et al., 2001). The imagers on polar orbits currently provide the only means to examine the precipitation characteristics on global scales with high temporal resolution.

The auroral electron energy flux can also be directly measured by observing the electron energy spectra on lowaltitude satellites (e.g. Hardy et al., 1987; Newell et al., 1996). Direct measurements have the disadvantage that the energy spectra are obtained only on the spacecraft path, and therefore global precipitation patterns can be studied only statistically over longer time periods. One of the largest and most widely used statistical analyses of the electron precipitation was carried out by Hardy et al. (1987), who organized the precipitation characteristics by the $K_{p}$ index. Based on the Hardy et al. (1987) statistics, on average the electron precipitation: 1) occurs in the nightside oval, more poleward (equatorward) for $\left.K_{p}<2\left(K_{p}>5\right) ; 2\right)$ varies smoothly over longitude for small $K_{p}$, but appears as hot spots for increasing $\left.K_{p} ; 3\right)$ deposits $<1 \mathrm{mWm}^{-2}\left(>4 \mathrm{mWm}^{-2}\right)$ on average at the oval for small (large) $K_{p}$. Similarly, the $A E$ index has been used to characterize the precipitation power. For example, Østgaard et al. (2002a) fitted the electron precipitation power results obtained by Østgaard et al. (2002b) using the $A E$ index in order to provide an estimate of the auroral flux.

Slinker et al. (1999) were the first to use a global MHD simulation for studying the electron precipitation characteristics. They found generally lower deposition rates as compared to estimates from global imagers and the Defense Meteorological Satellite Program (DMSP) spacecraft. Later, Palmroth et al. (2004a) studied the ionospheric total energy dissipation including both Joule heating and electron precipitation during a storm and a relatively minor substorm using another global MHD simulation (Grand Unified Magnetosphere Ionosphere Coupling Simulation, GUMICS-4). They found that in the simulation the temporal variation of the total integrated precipitation power follows that of the $A E$ index. The level of total integrated precipitation power in the simulation ionosphere was found to be lower than the Østgaard et al. (2002a) proxy. As similar conclusions applied to the total integrated Joule heating, Palmroth et al. (2004a) fitted the total ionospheric dissipation $P_{\text {ionosphere }}$ (Joule heating plus precipitation) computed from the simulation to the solar wind density $\rho$, velocity $v$, and the interplanetary magnetic field (IMF) $z$ component using a power law dependence, given by

$P_{\text {ionosphere }}=C\left(\frac{\rho}{\rho_{0}}\right)^{a}\left(\frac{v}{v_{0}}\right)^{b}\left[\exp \left(\frac{B_{z, I M F}}{\sqrt{2 \mu_{0} p_{d y n}}}\right)\right]^{d}$,

where $p_{d y n}=\rho v^{2}$ is the solar wind dynamic pressure. The fitting yielded similar values $(\sim 0.8, \sim 2.8$, and $\sim-2)$ for the exponents $a, b$, and $d$, respectively, in different types of events; and $\rho_{0}=m_{p} \cdot 7.3 \cdot 10^{6} \mathrm{~m}^{-3}=1.22 \cdot 10^{-20} \mathrm{kgm}^{-3}$ and $v_{0}=400 \mathrm{~km} / \mathrm{s}$ were chosen as typical solar wind density and velocity to obtain units of Watts for the constant $C$. As the right-hand-side of Eq. (1) agreed with the simulation results with more than an $80 \%$ correlation coefficient and, on the other hand, as both components of $P_{\text {ionosphere }}$ were independently in temporal agreement with $A E$-proxies, Palmroth et al. (2004a, b) argued that Eq. (1) can roughly predict the temporal behavior of ionospheric power dissipation as determined by the $A E$-proxies. It was also argued that if one scaled the GUMICS-computed total ionospheric power consumption to correspond with observational values, i.e., if Eq. (1) was "calibrated", it could predict the ionospheric power consumption correctly, both temporally and in magnitude. Hence, the power law might find use even in space weather forecasting.

The calibration of Eq. (1) includes two subjects: determining the capability of GUMICS-4 to reproduce ionospheric Joule heating and electron precipitation. The calibration concerning Joule heating was successfully carried out earlier (Palmroth et al., 2005). This paper has two scientific objectives: 1) to compare the precipitation morphology and energy characteristics computed from the GUMICS-4 simulation with direct measurements of auroral energy flux, and 2) to complete the calibration of Eq. (1). We simulate one 
event and compute the precipitation power in the simulation ionosphere. On the other hand, the total integrated auroral power is estimated using the global ultraviolet imager (UVI) onboard the Polar satellite. Independent of the Polar measurements, we compute the average auroral power using nitric oxide (NO) density measurements onboard the Student Nitric Oxide Experiment (SNOE) satellite. Furthermore, we compare the simulation results to the statistics of auroral power using the Hardy et al. (1987) results. The paper is organized as follows: Sect. 2 introduces the measuring techniques of auroral power as well as the GUMICS-4 global MHD simulation. Section 3 describes the simulated event as seen from the solar wind measurements and ground magnetometers. Section 4 presents the results of the total integrated auroral power, spatial distribution of the auroral energy flux, and the average of the auroral energy flux using the global imagers and SNOE measurements. Finally, Sect. 5 completes the paper with our discussion and conclusions.

\section{Assessing auroral power: techniques and models}

\subsection{Global imagers}

In the upper atmosphere, precipitating magnetospheric electrons excite the dominant neutral species, i.e., atomic oxygen and molecular nitrogen, which then emit auroral light as the atoms and molecules return to their ground states. At UV wavelengths the auroral emissions can be globally photographed with satellite UV imagers, such as that onboard the Polar spacecraft (Torr et al., 1995). Along with two oxygen lines, UVI measures two molecular nitrogen lines in the Lyman-Birge-Hopfield (LBH) bands centered approximately at $150 \mathrm{~nm}(\mathrm{LBHs})$ and $170 \mathrm{~nm}(\mathrm{LBHl})$. Since the emission in the LBH bands is due solely to electron impact excitation, in the absence of solar EUV flux the spectral brightness in the UVI image for LBH bands gives direct information of the incident electron flux (provided that there is no UV absorption between the emission altitude and the observing spacecraft). In reality, some absorption exists, and the main absorption mechanism is the Schumann-Runge absorption by $\mathrm{O}_{2}$, which is significant at altitudes below $\sim 150 \mathrm{~km}$ where $\mathrm{O}_{2}$ is abundant, and therefore it affects only the emission of higher energy electrons (e.g. emission in LBHs band) reaching this altitude. The longer wavelength and lower energy LBHl emission is virtually independent of the SchumannRunge absorption, and therefore the intensity of the LBHl emission is used in determining the energy flux of precipitating electrons. On the other hand, the ratio of the altitudedependent LBHs emission to LBHl emission in simultaneous images gives information concerning the mean energy of the incident flux. As both the LBHs and LBHl emission originate from nitrogen emissions, their ratio does not depend on compositional changes in the atmosphere (Germany et al., 1998).

\subsection{Nitric oxide measurements}

Along with the auroral emissions, the electron precipitation causes production of NO in the thermosphere. The lifetime of the auroral region NO is $\sim 1$ day (Barth et al., 2001), and therefore the NO produced by the nightside electron precipitation can be monitored in the dayside by an instrument observing the fluorescence of NO caused by the UV sunlight. Such an instrument is the Ultraviolet Spectrometer (UVS) onboard the Student Nitric Oxide Explorer (SNOE) satellite. Barth et al. (2004) reported on a method (hereafter referred to as the Barth method) with which the electron precipitation necessary to produce the NO density observed by SNOE can be calculated by using a time-dependent thermospheric NO model (Bailey et al., 2002) including neutral and ion photochemistry, and vertical diffusion. The model assumes that electron precipitation having a mean energy of $4 \mathrm{keV}$ takes place in the nightside (18:00-06:00 MLT). The calculation is iterative: first an estimate of nightside electron flux is compared to the observed daily average of the NO density, after which the ratio of the observation to the estimate is used to update the estimate. The iteration is typically carried out 5 times to obtain the electron flux that departs from the observed NO density by less than $1 \%$.

Because the major loss mechanism of NO is photodissociation occurring in the dayside thermosphere, most of the NO produced by the nightside electron precipitation decays only after the Earth rotates to bring the location of production to the dayside. Throughout the dark sector, the location of production gains more $\mathrm{NO}$ as long as electron precipitation is taking place. Thus, the SNOE observation of the $\mathrm{NO}$ is essentially an integration of the NO production that has been taken place on the nightside. Zonal thermospheric winds transfer NO from the location of production to some extent, but do not severely affect the measurements, because the speeds of these winds are typically only a few meters per second at auroral latitudes (Fuller-Rowell, 1995). As the time constant of decay of NO is $\sim 1$ day, the $\sim 12 \mathrm{~h}$ in sunshine is not enough to totally clear out the thermosphere of NO, and hence some of the measured nitric oxide may have been produced the night before or even earlier. However, as the Barth method estimates the electron precipitation necessary to explain the production of NO by comparing to the daily average, this effect should be taken into account.

\subsection{GUMICS-4 global MHD simulation}

GUMICS-4 (Janhunen, 1996) is a global MHD simulation, which solves the ideal MHD equations in conservative form in the solar wind and in the magnetosphere. The MHD simulation box in the $X_{G S E}$ direction reaches from $32 R_{E}$ to $-224 R_{E}$, the $Y_{G S E}$ and $Z_{G S E}$ directions reach $\pm 64 R_{E}$. In the near-Earth region the MHD simulation box is limited by a $3.7 R_{E}$ spherical shell, which maps along the dipole field to approximately $60^{\circ}$ in magnetic latitude. The grid in the 


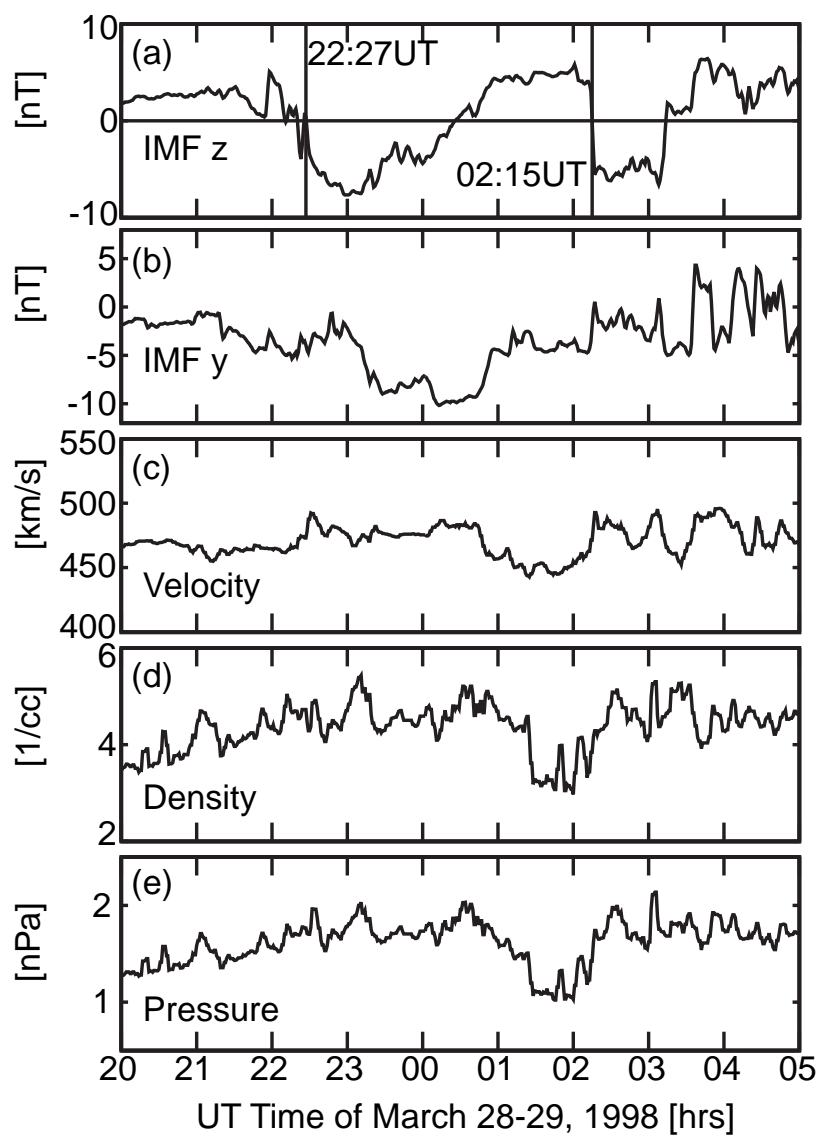

Fig. 1. Solar wind conditions on 28-29 March 1998, as recorded by the Wind satellite: (a) IMF $z$, (b) IMF $y$, (c) solar wind velocity, (d) proton density, and (e) solar wind dynamic pressure; all in Geocentric Solar Ecliptic (GSE) coordinate system.

MHD simulation box is a Cartesian octogrid, and it is adaptive meaning that when the code detects large spatial gradients, the grid is refined. Solar wind input variables (temperature, density, velocity and magnetic field) are treated as boundary conditions in the sunward wall of the simulation box; outflow (Neumann) boundary conditions are applied on other walls of the simulation box. The internal geomagnetic field is modeled by a dipole placed at the center of the Earth.

The MHD magnetosphere is coupled to a high-resolution ionosphere using electrostatic current continuity equations. The ionosphere is a spherical shell at an altitude of $110 \mathrm{~km}$, and the region between the ionosphere and the $3.7 R_{E}$ shell is a passive medium, which only transmits electric effects, and where no currents flow perpendicular to the magnetic field. A triangular finite element grid of the ionosphere is fixed in time, although refined to $\sim 100-\mathrm{km}$ spacing in the auroral oval region. The magnetosphere delivers fieldaligned currents and electron precipitation to the ionosphere. The precipitating electrons are assumed to originate from a Maxwellian source population having the magnetospheric density and temperature; and a finite probability to fall into the loss cone. The equation for the electron precipitation energy flux $\epsilon_{\text {prec }}$, assuming that the parallel potential drop is zero, is given by

$\epsilon_{\mathrm{prec}}=\sqrt{\frac{2}{\pi m_{e}}} n_{e} T_{e}^{3 / 2}$,

where $m_{e}$ is the electron mass and the electron temperature $T_{e}$ is calculated from the ion temperature $T_{i}$ of the MHD fluid by assuming $T_{e}=T_{i} / 4$. The ionospheric calculation further includes the determination of Pedersen and Hall conductivities from electron density modulated by the precipitation and solar EUV radiation. Together with the conductivities, the field-aligned currents determine the ionospheric electric potential, which is mapped back to the magnetosphere, where it is used as a boundary condition for the MHD equations. Further information on the code structure and performance can be found, e.g. in Palmroth et al. (2001; 2004a).

\section{28-29 March 1998: event description}

The Wind spacecraft, located at $(230,-22,-6) R_{E}$ in geocentric solar ecliptic (GSE) coordinate system, recorded a southward turning of the IMF at 22:27 UT after several hours of northward orientation (Fig. 1a). The IMF $B_{z}$ rotated relatively smoothly from north to south reaching about $-8 \mathrm{nT}$ at $\sim$ 23:00 UT, after which the northward orientation was attained at 00:27 UT on 29 March 1998. Another southward turning at 02:15 UT was followed by a northward turning an hour later, at 03:14 UT. The IMF $B_{y}$ (Fig. 1b) fluctuated between zero and $-10 \mathrm{nT}$ during the two southward orientations. Solar wind velocity (Fig. 1c) varied between 450 and $500 \mathrm{~km} / \mathrm{s}$ throughout the time interval of interest, whereas the solar wind density (Fig. 1d) remained in the range $4-5 \mathrm{~cm}^{-3}$. The solar wind dynamic pressure (Fig. 1e) fluctuated between 1 and $2 \mathrm{nPa}$. The time delay between the Wind $X_{G S E}$ position and the magnetopause is $\sim 49 \mathrm{~min}$ using the average of the solar wind velocity in $X_{G S E}$ direction $(\sim 475 \mathrm{~km} / \mathrm{s})$.

The solar wind conditions led to the development of two successive substorms. At 23:45 UT the Nuuk (GHB) magnetometer station located at the west coast of Greenland ( 21:15 MLT; $70.5^{\circ}$ magnetic latitude) recorded a northward propagating negative bay in the $H$ component, 29 minutes after the southward IMF had arrived at the magnetopause (Fig. 2). The onset timing was corroborated with an auroral intensification recorded by the global imagers onboard the Polar satellite. Another clear northward propagating negative bay in the $H$ component was recorded at $\sim 04: 00$ UT by the Narsarsuaq (NAQ) station on the west coast of Greenland (02:00 MLT; $66.3^{\circ}$ magnetic latitude), and again the timing is supported by the data recorded by the global imagers onboard Polar. As characterized by the $A E$ index (Fig. 2), the 
first intensification reached almost $1000 \mathrm{nT}$, whereas the second was $\sim 500 \mathrm{nT}$ in magnitude. The two events were accompanied by tail field dipolarizations and particle injections at the geosynchronous orbit (see Palmroth et al., 2005).

\section{Results}

\subsection{Spatial distribution}

The first row of Fig. 3 presents the spatial distribution of the auroral luminosity as measured by the UVI instrument (with the LBHl filter) onboard the Polar spacecraft. Since auroral power is calculated fromUVI images, the spatial distribution of auroral luminosity is proportional to the auroral power. The second row of Fig. 3 gives the auroral power as computed using the Hardy et al. (1987) statistical model for $K_{p}$ values 3+ and 5-, respectively, whereas the third row presents the auroral power in the Northern Hemisphere in the GUMICS-4 global MHD simulation. The first column represents a time slightly after the first onset (23:50 UT), whereas the second column is the time of the first substorm maximum (01:30 UT).

The onset of the first substorm occurs in the premidnight sector as shown by the UVI image at 23:50 UT. Naturally a statistical model cannot produce precipitation patterns indicative of a substorm sequence; however here the Hardy et al. (1987) model serves as an example of the amount of typical auroral powers expected during times of $K_{p} 3+$. As shown by the GUMICS-4 results at 23:50 UT, the enhancement of the precipitation power coincides with the enhancement of the auroral luminosity as measured by the UVI instrument, indicating that the GUMICS-4 simulation predicts the location of the substorm onset correctly in this event. The maximum GUMICS-4 precipitation power in the Northern Hemisphere is $\sim 1.5 \mathrm{mWm}^{-2}$. The Hardy et al. (1987) model indicates $\sim 2 \mathrm{mWm}^{-2}$ throughout the oval, whereas in most parts of the nightside oval the average of GUMICS4 precipitation power is $\sim 0.6 \mathrm{mWm}^{-2}$. This suggests that the GUMICS-4 precipitation power in the Northern Hemisphere during the onset of the first substorm presumably is too low (although the spatial distribution has similarities with the UVI measurements).

At the maximum of the first substorm (01:30 UT), the premidnight sector still shows the maximum auroral luminosity, although the luminosity is spread throughout the oval. The Hardy et al. (1987) statistical model at $K_{p}$ 5- shows enhanced precipitation in the premidnight and morning sectors, consistent with the most likely locations of electron acceleration events during southward IMF (Newell et al., 1996). Compared to the time of the onset at 01:30 UT, the GUMICS4 results show small warm spots in the vicinity of the dusk terminator and the cusp. A small spot of enhanced luminosity in the dusk terminator can also be seen in the UVI image. Hence, the spatial distribution of GUMICS-4 precip-

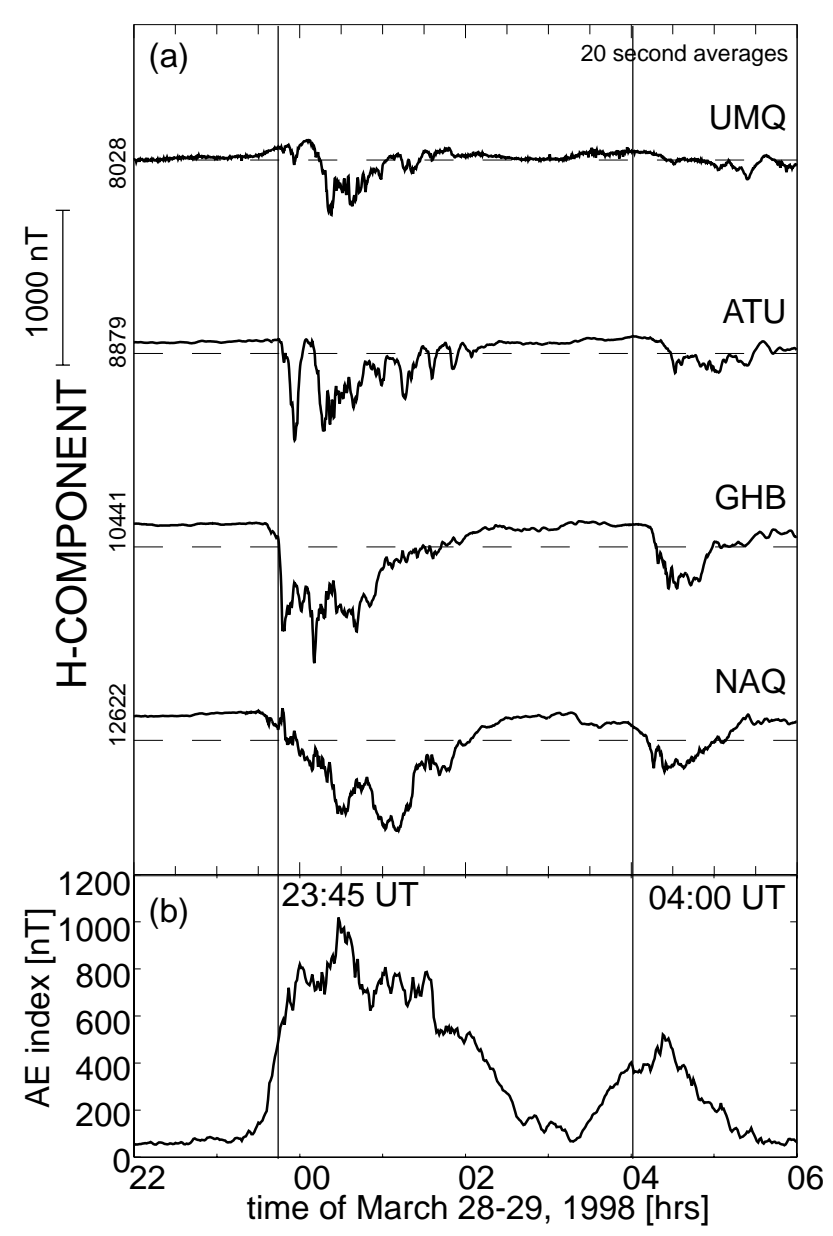

Fig. 2. (a) Ground magnetic field $H$ component of the Greenland West Coast magnetometer stations UMQ (northernmost in this plot), ATU, GHB, and NAQ (southernmost). (b) $A E$-index.

itation again has similarities with the UVI image and Hardy et al. (1987) statistics. However, we again see that the level of GUMICS-4 precipitation is lower than suggested by the Hardy et al. (1987) statistics.

\subsection{Total auroral power}

GUMICS-4 power estimates are compared with UVI observations in Fig. 4. UVI power was calculated using three independent calculations based on the methods previously reported by Germany (Germany et al., 1998; 2004), Lummerzheim (Lummerzheim et al., 1991), and Liou (Liou et al., 2001). Multiple calculations were used in recognition of the fact that there are differences in the processing algorithms used by different researchers. Taken together, the different calculations represent a range of expected power values that can be compared with the GUMICS-4 estimates.

The red, green and blue traces in Fig. 4a show the total auroral power observed by UVI on 28-29 March 1998 in 
$23: 50 \mathrm{UT}$

$28 \operatorname{Mar} 98$
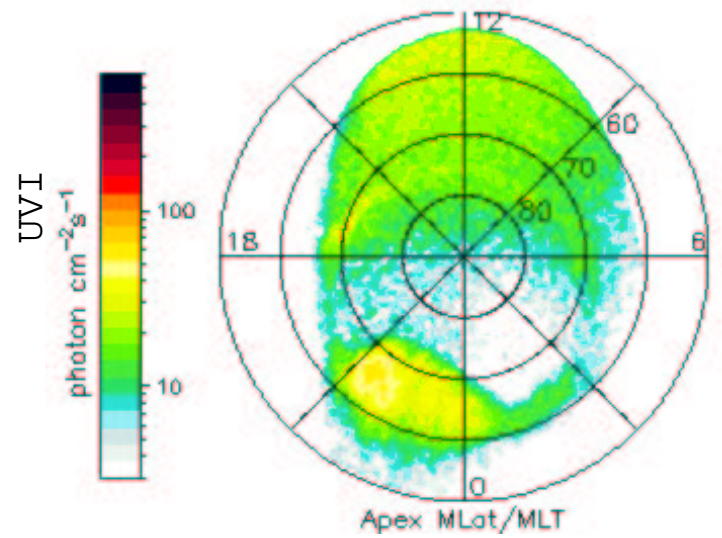

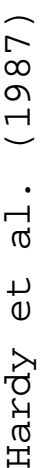

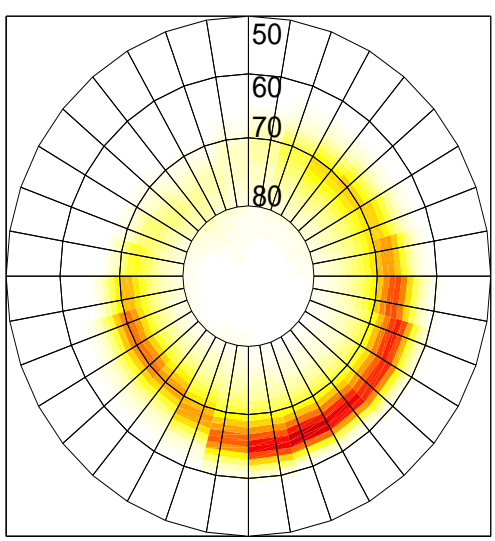

$\mathrm{Kp}=3+$

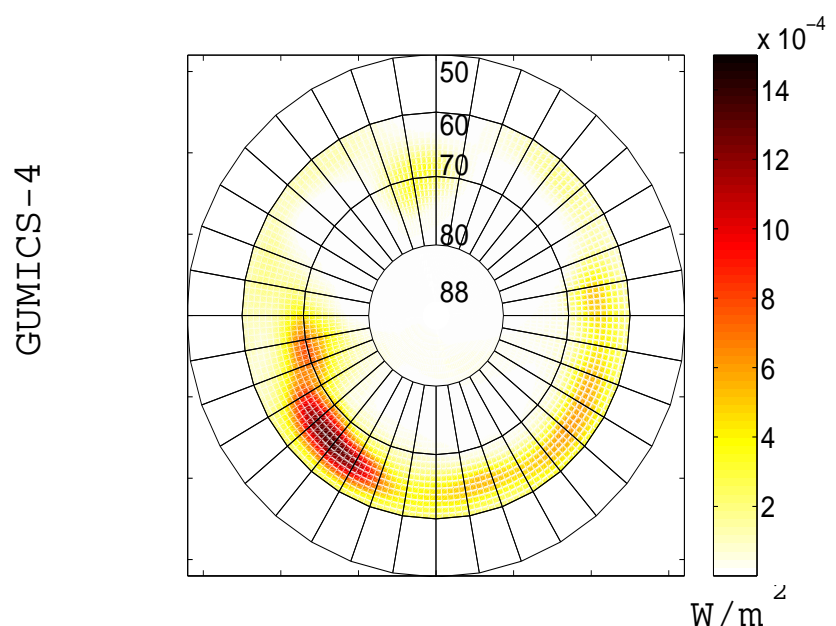

$01: 30 \mathrm{UT}$

\section{Mar $98 \quad 01: 29: 41$ UT}
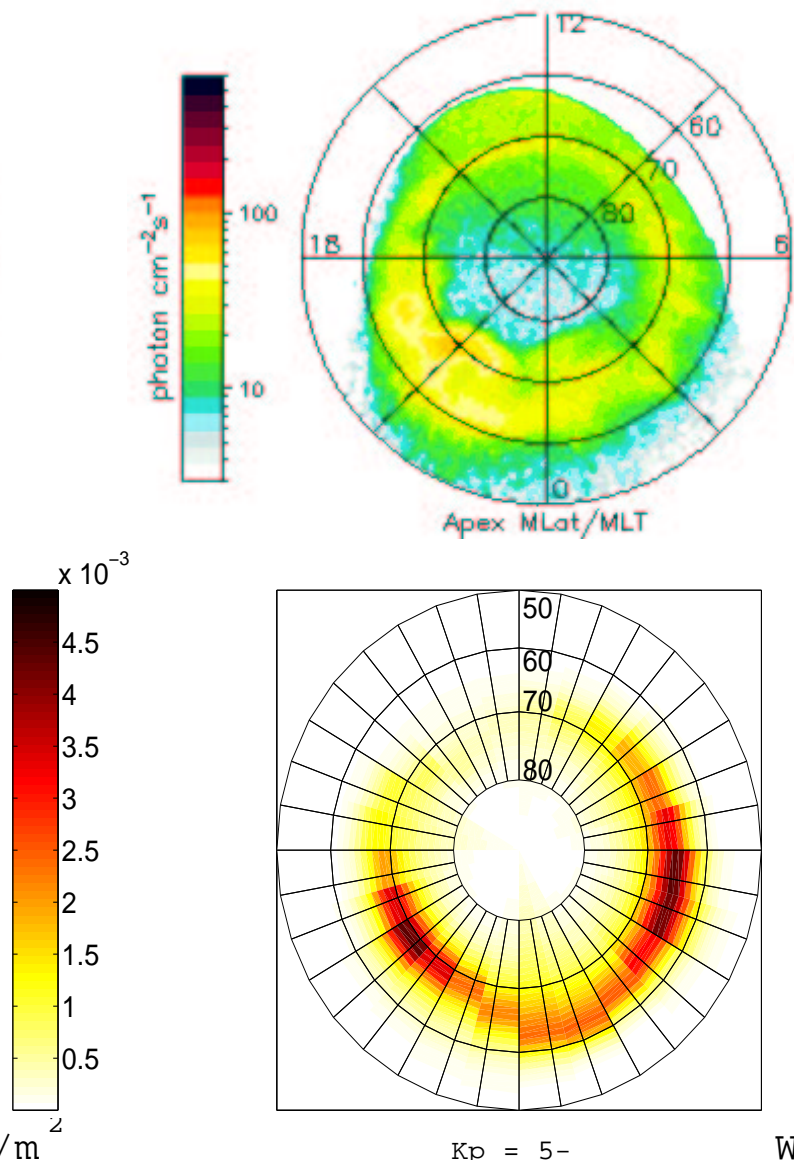

$\mathrm{Kp}=5-$
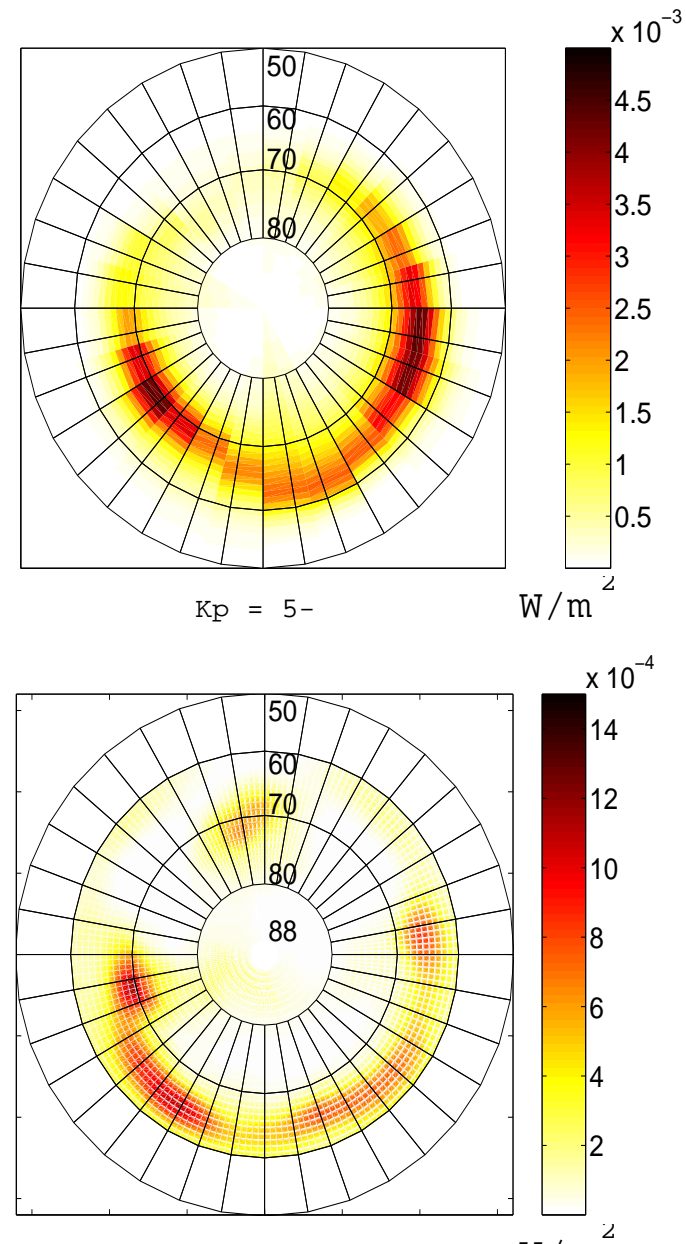

$\mathrm{W} / \mathrm{m}^{2}$

Fig. 3. Spatial distribution of auroral luminosity in UVI (first row), and auroral power in Hardy et al. (1987) for $K_{p} 3+$ and $5-$, respectively (second row), and GUMICS-4 global MHD simulation (third row) for slightly after the onset (first column, 23:50 UT) and for the maximum of the first substorm (second column, 01:30 UT). Notice that the color scales do not match row-wise. 
the Northern Hemisphere as determined by the methods of Germany, Lummerzheim, and Liou, respectively. (This can be used as an estimate of the total hemispheric power if the camera is viewing the full auroral oval.) The black trace in Fig. $4 \mathrm{a}$ is the auroral power computed using the Hardy et al. (1987) statistical model. Although organized using the $K_{p}$ index and therefore having a poor temporal resolution, the Hardy et al. (1987) model gives the auroral power based on an extensive statistical survey from the DMSP satellite recordings.

The three UVI power estimates capture the temporal evolution of the event and show significantly greater power deposition than that shown by the Hardy et al. (1987) statistical model. The differences with the Hardy et al. (1987) model illustrate the variability that can be expected between single events and stastical averages of many events. They also illustrate the value of auroral observations over statistical models for single event studies.

Surprisingly, the three methods disagree significantly on the magnitude of the power deposited during the active periods. This is probably due to differences in the conversion methods from photon brightnesses to total power. The Germany and Lummerzheim calculations use fixed conversions based on the pre-flight calibrations of the UVI instrument while the Liou calculation uses an empirical value fixed to DMSP electron measurements (Carbary et al., 2004). Other, smaller, differences are probably due to differences in selection of auroral regions to contribute to the total power and in the determination of the non-auroral airglow contribution to the total LBH brightness. These differences are of interest, but are not the focus of this paper. For the present purposes, they are sufficient to provide a range of values for comparison with GUMICS-4.

The black trace in Fig. $4 \mathrm{~b}$ is the Northern Hemisphere integrated auroral power computed using Eq. (2) in the GUMICS-4 simulation of the 28-29 March 1998 event; the dashed line indicates the Southern Hemisphere precipitation power. The integration is limited to poleward of $60^{\circ}$ because the inner edge of the magnetospheric simulation $\left(3.7 R_{E}\right)$ maps to this latitude and therefore there cannot be precipitation of magnetospheric origin equatorward of this latitude. The auroral power in the Northern Hemisphere in Fig. 4b computed using GUMICS-4 simulation does not show the temporal variation indicated by the other traces in Fig. 4a. This is atypical; in simulations of other events the temporal variation of precipitation power has been similar to that of the $A E$ index (e.g. Palmroth et al., 2004a). However, the GUMICS-4 Southern Hemisphere precipitation manifests some of the temporal variation of the event as there is a slight increase in the power during the substorm maxima. This is likely a coincidence as the Southern Hemisphere precipitation is altogether at a higher level during this event: the Southern Hemisphere shows about $60 \%$ larger values as compared to those obtained from the Northern Hemisphere. The amount of precipitation power in the Northern

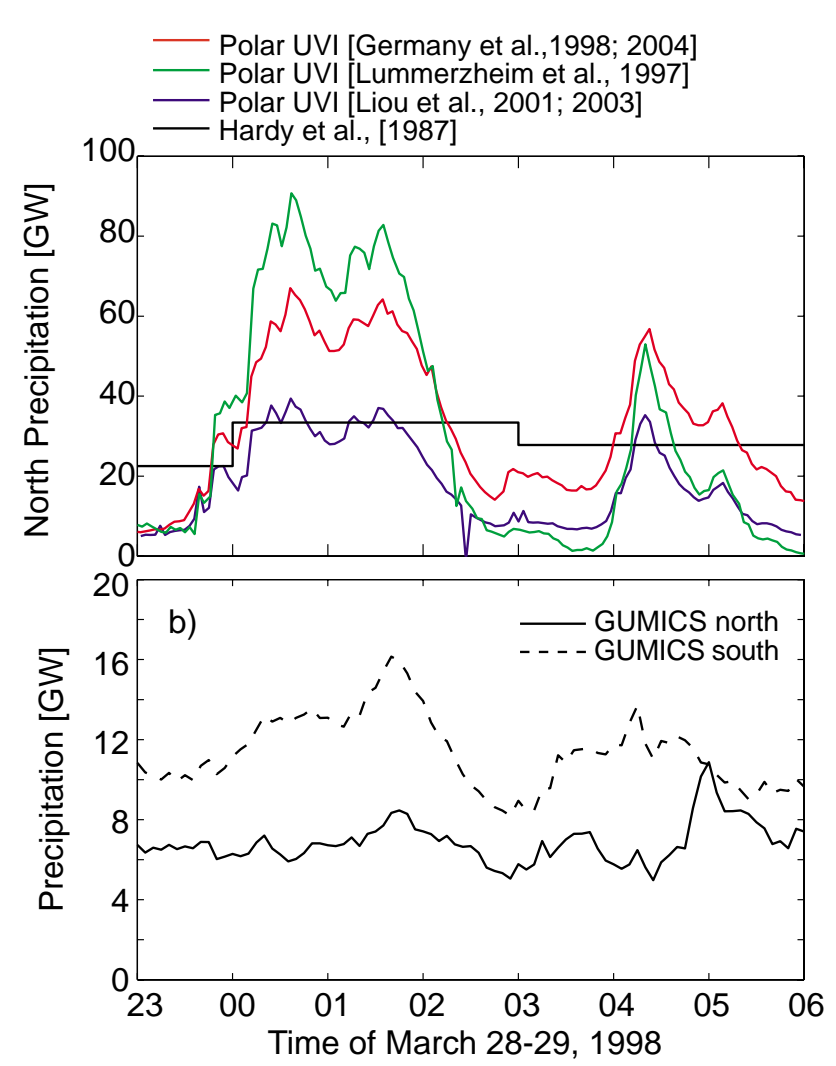

Fig. 4. (a)Total Northern Hemisphere precipitation power as computed by the Germany method (red), Lummerzheim method (green), Liou method (blue), and Hardy et al. (1987) statistical method (black). (b) North (solid) and south (dashed) total integrated precipitation power in GUMICS-4 simulation.

Hemisphere in GUMICS-4 resembles that of the quiet times as both the Liou and Lummerzheim methods are in quantitative agreement with GUMICS-4 results before the two intensifications. During the intensifications the magnitude of GUMICS-4 auroral power is about 3 times lower than that of the Liou method.

\subsection{Average precipitation at auroral latitudes}

In order to obtain a measure of auroral power completely independent of the methods based on global imagers onboard Polar spacecraft, we investigate the nightside precipitation power also using the SNOE satellite NO measurements. As was noted in Sect. 2.2, the Barth method computes essentially the integrated nightside precipitation using the SNOE satellite recordings. If normalized with the area in which the measurement is carried out, one should be left with a fairly good estimate of the average nightside precipitation. 


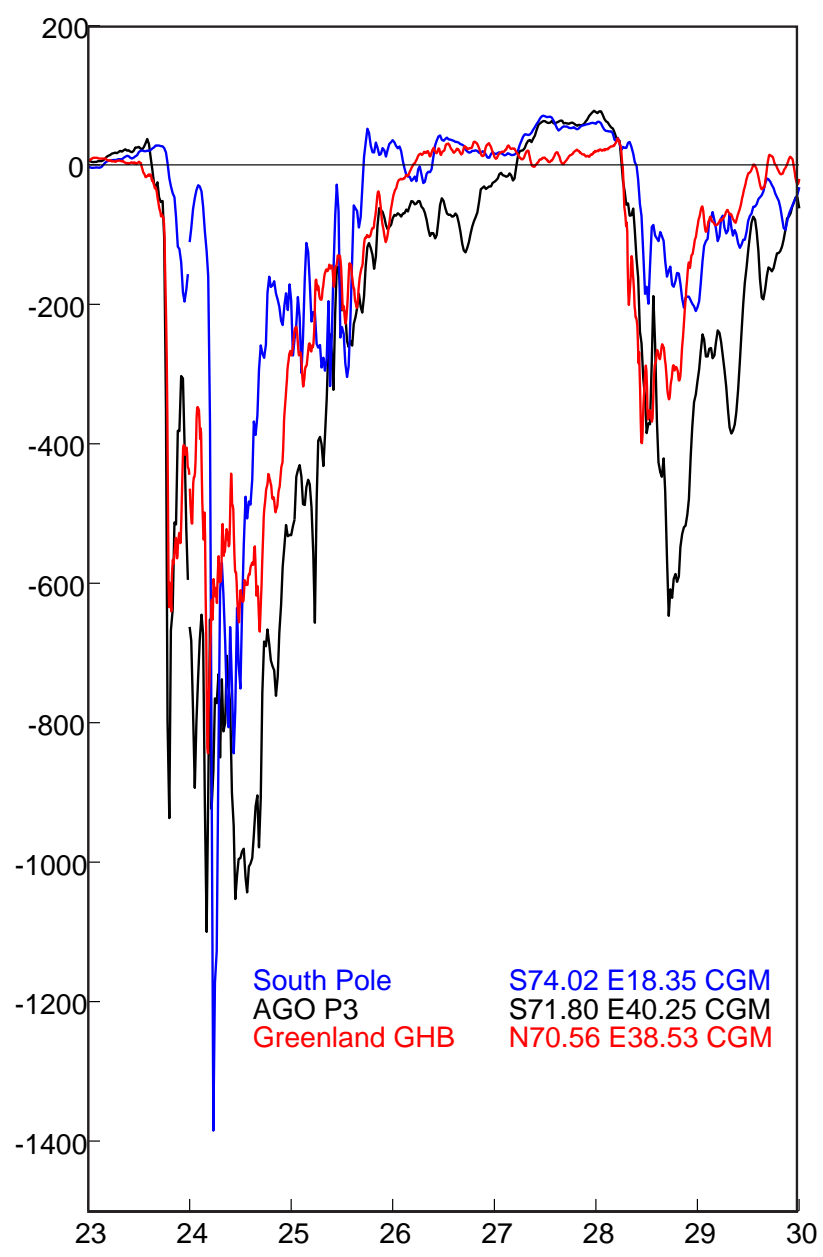

Fig. 5. $H$-component $[\mathrm{nT}]$ as recorded by the South Pole (blue) and AGO P3 (black) stations on the Southern Hemisphere. (red) $\mathrm{H}$ component as recorded by the Greenland Nuuk station. The magnetometer data are shifted to start from zero to allow comparison in the intensities.

Therefore, in this section we investigate the outcome of

$P_{P R 65^{\circ}}=\frac{\int_{62.5^{\circ}}^{67.5^{\circ}} \epsilon d S}{\int_{62.5^{\circ}}^{67.5^{\circ}} d S}$,

where $\epsilon$ is the energy flux of precipitating electrons, and the integration is carried out in a 5-deg latitude band centered at $65^{\circ}$ magnetic latitude in the nightside. The outcome of the analysis in Eq. (3) can also be computed from the GUMICS4 simulation and from the precipitation energy data based on global imagers. Furthermore, Fig. 3 indicates that the maximum of auroral emission occurs at $65^{\circ}$ both in GUMICS-4 and in UVI images, and thus the choice of latitude in the computation is justified.

As the SNOE measures NO once per orbit 15 times a day, a spatial distribution of precipitation power cannot be
Table 1. Average of Eq. (3) over the substorm sequence.

\begin{tabular}{lll}
\hline Method & $<$ Eq. $(3)>\left[\mathrm{mW} / \mathrm{m}^{2}\right]$ & $<$ Eq. $(3)>\left[\mathrm{mW} / \mathrm{m}^{2}\right]$ \\
& Northern Hemisph. & Southern Hemisph. \\
\hline Germany & 2.6 & - \\
Lummerzheim & 2.2 & - \\
Hardy et al. (1987) & 2.0 & 2.0 \\
Barth & 0.6 & 0.9 \\
Liou & 0.5 & - \\
GUMICS-4 & 0.5 & 0.7 \\
\hline
\end{tabular}

obtained globally with the same temporal resolution as using the global imagers. Furthermore, as an integration of the nightside precipitation, the outcome of the Barth method does not specify in which location the precipitation originally took place. Therefore, the spatial variation must be averaged out from the SNOE recordings by, e.g. taking a time average over the 6-h substorm sequence. Table 1 lists the time average of Eq. (3) over the substorm sequence computed from the precipitation power data obtained from the Polar spacecraft global imagers, Hardy et al. (1987) statistics, SNOE measurements, and the GUMICS-4 simulation. Essentially, an entry in Table 1 is the average nightside precipitation at $65^{\circ}$ during the entire period of interest. The Germany and Lummerzheim methods and the Hardy et al. (1987) statistics all yield comparable values, while Liou and Barth methods yield values comparable to the GUMICS-4 simulation. The Hardy et al. (1987) statistical method yields a surprisingly large value considering that the total integrated power from the Hardy statistics is much lower than that, e.g. from the Lummerzheim method. This is because in the Hardy et al. (1987) method the nightside precipitation maximizes in the vicinity of $65^{\circ}$ and it has little variability in longitude (cf. Fig. 3), while during substorms the precipitation should have larger variability in longitude.

Table 1 shows an estimate of the Southern Hemisphere precipitation power as computed by the Barth method and the GUMICS-4 simulation. Although the event took place at the equinox and the dipole tilt angle increases from $0^{\circ}$ to $6^{\circ}$ during the substorm sequence, both the Barth method and GUMICS-4 simulation show a hemispherical asymmetry in the precipitation power, with more power deposited in the Southern Hemisphere. Figure 5 showing ground magnetometer recordings on nearly conjugate locations on Northern and Southern Hemisphere indicates that the substorm is indeed more intense in the Southern Hemisphere. Namely, the $H$-component of the GHB station (red trace in Fig. 5) consistently indicates weaker ground magnetic field perturbations than on the AGO P3 station (black trace in Fig. 5) on Southern Hemisphere. For reference, Fig. 5 shows also the magnetic recordings of the South Pole station (blue), slightly poleward of the AGO P3 station. The Southern Hemisphere 
recordings are shifted to have the same base line with the GHB recordings. The substorm features in the GHB and AGO P3 recordings occur precisely at the same time throughout the plotted period, indicating that it is reasonable to believe in a fairly good magnetic conjugacy. Although the cause of the stronger substorm in the Southern Hemisphere is not clear, Fig. 5 suggests that the noted asymmetry in hemispheric power in Table 1 may be real (and not an artifact of the methods).

\section{Discussion}

In this paper, we have compared the precipitation power results from the GUMICS-4 global MHD simulation to observational data. Aiming to calibrate Eq. (1), the purpose of the study is to determine whether the GUMICS-4 simulation is able to predict the total ionospheric power deposition. The calibration concerning the larger ionospheric energy sink, the Joule heating, was successfully carried out earlier (Palmroth et al., 2005). As far as precipitation is concerned, the calibration includes three questions: Is the GUMICS-4 precipitation well-reproduced in terms of 1) spatial variation, 2) temporal variation, and 3) the magnitude of the integrated hemispheric power.

\subsection{Observations}

Given that the process of computing the hemispheric power from the UVI images includes errors due to dayglow removal, slant path removal, instrument calibration, and the value used to convert the image brightness into precipitation power, it is not surprising that the three methods do not agree to the last digit. Out of the mentioned error sources, the dayglow and the slant path removal probably introduce smaller errors between the three methods, and the instrument calibration is naturally similar as all the methods use the same data. For converting the image brightness into precipitation power, the Liou method uses an empirical value fixed to DMSP electron measurements (Carbary et al., 2004), while the Lummerzheim and Germany methods use a fixed value (typically $150 \mathrm{R} / \mathrm{mWm}^{-2}$ and $120 \mathrm{R} / \mathrm{mWm}^{-2}$, respectively).

For the sake of calibration of Eq. (1), our difficult task is to determine the most probable magnitude of hemispheric power. Basically, the observational results can be summarized in the following way: The methods agree as far as temporal variation is concerned. Likewise, the methods agree that the quiet-time hemispheric power is about $10 \mathrm{GW}$. However, during the activations the methods do not agree, which brings ambiguity to the value needed to calibrate the GUMICS- 4 results. Two of the three methods (Lummerzheim and Germany) agree that the integrated power is more than $40 \mathrm{GW}$ during the two intensifications. Although the SNOE measurement (Table 1) supports the lower power deposition rates, the Germany and Lummerzheim methods yield values that are closer to the Hardy et al. (1987) statistics, which is to be taken as average value of precipitation due to the $K_{p}$ dependency. Given that the SNOE value in Table 1 may be an underestimation due to the lower spatial coverage and heavy time averaging, we find that the following compromise for the integrated auroral power yields a result that agrees rather well with the observations: The quiet-time value is about $10 \mathrm{GW}$, while the active-time value is given by a simple average of the three methods. During quiet times, this compromise is in accordance with the SNOE observations, because the Barth method agrees with the Liou method (Table 1). During the activations, the average of the Polar UVI methods is close to the values given by the Germany method. The peak power during the substorms is then $\sim 50 \mathrm{GW}$, while the quiet time value before the two intesifications is $\sim 10 \mathrm{GW}$.

\subsection{Comparison of GUMICS-4 to observations}

GUMICS-4 seems to reproduce the spatial variation of the Northern Hemisphere precipitation reliably in the sense that locations of the brightest luminosity in UVI are also visible in the GUMICS-4 results during this event. Furthermore in the GUMICS-4 simulation, the onset of the first substorm occurs at the right time in the roughly correct location (the $\sim$ 21:00 MLT hot spot). However, in GUMICS-4 during this event, the precipitation in the Northern Hemisphere appears in hot spots and varies such that at times some spots brighten while others fade. The compensation of simultaneous brightenings and fadings leads to a flat total integral in the Northern Hemisphere, while some similarities with the Polar measurements are observed in the total integral of the Southern Hemisphere precipitation. Earlier results of a small substorm and a major storm show that the temporal behavior of the Northern Hemisphere precipitation is atypical, as the total integrated precipitation from the Northern Hemisphere have agreed with the $A E$ index before (Palmroth et al., 2004a).

The reason for the lack of temporal variation in the Northern Hemisphere during the present event is difficult to explain as (with the exception of the sunward boundary conditions) the code setup used in the present event is identical with the one presented in Palmroth et al. (2004a). However, the precipitation during intense substorms (such as the present event) is closely related with reconnection and explosive loading-unloading processes releasing magnetic energy in the tail. On the other hand, the ideal MHD cannot de facto reproduce as efficient reconnection as, for example, the Hall-MHD or hybrid codes (e.g. Birn et al., 2001). Therefore, one should rather ask why should the precipitation results in a global MHD simulation show the temporal variation of processes (such as reconnection) that are not properly included in the original ideal MHD theory. In fact, considering these issues, the GUMICS-4 results seem rather positive, as the magnitude of the precipitation is in quantitative agreement with the SNOE and Polar UVI measurements during 


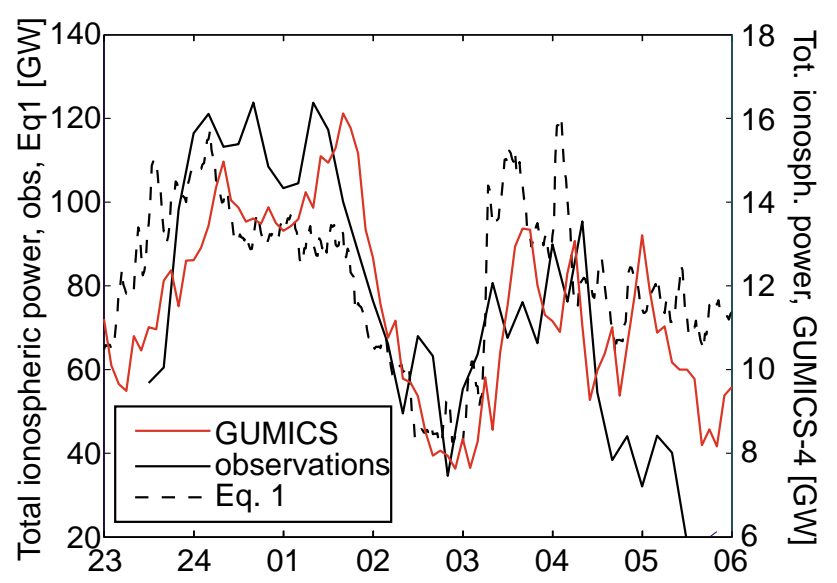

Fig. 6. Northern Hemispheric total power (Joule heating plus precipitation) in GUMICS-4 (solid red), in observations (solid black), and from scaled Eq. (1) (black dashed). The observational Joule heating is computed using measurements from SuperDARN radars and Polar UVI (see details in Palmroth et al. (2005)), and precipitation is computed in this study.

quiet times before the two intensifications, and some of the temporal variation is seen on the Southern Hemisphere. We speculate that the better reproduction of the Southern Hemisphere temporal variation is a coincidence due to the fact that the Southern Hemisphere is altogether more intense during this event. As the experience has shown that the GUMICS4 simulation is more directly driven than it exhibits classical loading-unloading features, we speculate that the features visible (missing) in the GUMICS-4 results are due to the directly driven (loading-unloading) processes. This speculation is further supported by the fact that the good correlation between the $A E$ index and the precipitation in Palmroth et al. (2004a) was observed during a major storm and a weak substorm. Namely, storms in general may be more directly driven than substorms, whereas a weak substorm is likely associated with inefficient reconnection and therefore they may be more "reproducible" by the ideal MHD.

\subsection{Calibration}

Naturally, the failure of GUMICS-4 in reproducing the temporal variation of total integrated precipitation power in the Northern Hemisphere is disturbing while trying to quantitatively calibrate Eq. (1), particularly when the calibration associated with the Northern Hemispheric Joule heating was carried out successfully (Palmroth et al., 2005). Namely, the temporal variation of Joule heating in GUMICS-4 was in good accordance with the observed Joule heating and the $A E$ index. Furthermore, it was found that the magnitude of Joule heating was quite consistently a factor of 10 less than the observed hemispheric Joule heating. Therefore the multiplication of GUMICS-4 Joule heating by 10 would lead to at least a sufficiently representative result.

As can be seen from Fig. 4, the correlation between the GUMICS-4 Northern Hemisphere precipitation power and the observations is poor. However, Eq. (1) was developed for global dissipation including both hemispheres. If we take an average of GUMICS-4 global precipitation including both hemispheres, the correlation coefficient between the simulation precipitation results and the average Polar UVI observations improves to 0.7 . This average of global precipitation in the simulation is quite consistently 5 times less than the observations indicate during the active times, while during quiet times the GUMICS-4 result quantitatively agrees with the observations without any multiplication.

As discussed in Palmroth et al. (2005), the lower Joule heating rates in GUMICS-4 compared to the observations is mainly due to the weak Region 2 current system, as Joule heating is largely produced as the Region 1 and 2 currents close to each other through the highly conducting oval. The improper Region 2 system is a typical feature in global MHD simulations due to insufficient modeling of the inner magnetosphere. We speculate that better modeling of the inner magnetosphere (with finer grid sizing and/or going beyond ideal MHD) will improve the modeling of the Region 2 current system, and increase the amount of Joule heating in the model. As for the precipitation, it is shown here that the quiet-time observations are already in quantitative agreement with the GUMICS-4 results, indicating that ideal MHD is sufficient to model the diffuse aurora. The factor 5 difference between the observations and the GUMICS-4 results during active times is not a poor achievement, at least when considering that there are two- or threefold differences in the observational results themselves. However, we speculate that the active time difference between the GUMICS-4 results and the observations is probably due to insufficient modeling of the explosive reconnection processes in the tail, and the fact that the parallel potential drop is set to zero in GUMICS-4. The latter is a conscious choice as the parallel potential drop is associated with discrete arc physics, which deals with much smaller scale sizes than the smallest grid spacing in the GUMICS-4 ionosphere $(\sim 100 \mathrm{~km})$. Furthermore, it is not clear how the parallel potential drop should be added between the ionosphere and magnetosphere, as based on these results it appears that it should be "on" during auroral activations only.

As Joule heating is a larger sink compared to electron precipitation (Lu et al., 1998; Knipp et al., 1998), the temporal variation of precipitation does not largely affect the temporal variation of the total ionospheric power dissipation. Indeed, in the Northern Hemisphere, Fig. 6 indicates a good accordance between the Northern Hemisphere power dissipation in the simulation (Joule heating plus precipitation) and observations, where the observed total power is computed using measurements presented in this study as well as those in Palmroth et al. (2005). The correlation coefficient is 0.8 . Furthermore, the total ionospheric power in the simulation 
correlates with the outcome of Eq. (1) (dashed black trace), with 0.83 correlation coefficient. The outcome of Eq. (1) is computed using $a=0.8, b=2.8$, and $d=-2$, while the original scaling constant $C$ is multiplied to take into account the Joule heating and precipitation factors. The correlation coefficient between the $A E$ index and the GUMICS-4 total Northern Hemisphere power is 0.85 . Therefore, although the precipitation in the Northern Hemisphere does not follow the $A E$ index during this event, the total power dissipation in the simulation is rather well-correlated with the $A E$ index $(85 \%)$, observations (80\%), and the outcome of Eq. (1) (83\%). This indicates that the calibration has succeeded, and a representative value of global power dissipation during active times may be obtained from Eq. (1), if the hemispheric Joule heating is multiplied with 10 and the hemispheric precipitation with 5. During quiet times, the multiplication of hemispheric Joule heating by 10 is enough to produce a representative result of global ionospheric dissipation.

Acknowledgements. We gratefully acknowledge partial support from National Science Foundation Grants OPP-0338105 and OPP0341470. We further thank L. J. Lanzerotti and T. J. Rosenberg for magnetometer data interpretation and helpful discussions. The work of M. Palmroth is supported by the Academy of Finland.

Topical Editor M. Pinnock thanks a referee for her/his help in evaluating this paper.

\section{References}

Akasofu, S.-I.: Energy coupling between the solar wind and the magnetosphere, Space Sci. Rev., 28, 121-190, 1981.

Bailey, S. M., Barth, C. A., and Solomon, S. C.: A model of nitric oxide in the lower thermosphere, Journal of Geophys. Res., 107(A8), 1205, doi:10.1029/2001JA000258, 2002.

Baker, D. N., Turner, N. E., and Pulkkinen, T. I.: Energy transport and dissipation in the magnetosphere during geomagnetic storms, J. Atm. Solar Terr. Phys., 63, 421-429, 2001.

Barth, C. A., Baker, D. N., Mankoff, K. D., and Bailey, S. M.: The northern auroral region as observed in nitric oxide, Geophys. Res. Lett., 28, 1463-1466, 2001.

Barth, C. A., Baker, D. N., and Bailey, S. M.: Seasonal variation of auroral electron precipitation, Geophys. Res. Lett., 31, doi:10.1029/2003GL018892, 2004.

Birn, J., Drake, J. F., Shay, M. A., Rogers, B. N., Denton, R. E., Hesse, M., Kuznetsova, M., Ma, Z. W., Bhattacharjee, A., Otto, A., and Pritchett, P. L.: Geospace Environmental Modeling (GEM) magnetic reconnection challenge, J. Geophys. Res., 106, 3715-3720, 2001.

Carbary, J. F., Sotirelis, T., Newell, P. T., and Meng, C.-I.: Correlation of LBH intensities with precipitating particle energies, Geophys. Res. Lett., 31, L13801, doi:10.1029/2004GL019888, 2004.

Fuller-Rowell, T. J.: The dynamics of the lower thermosphere, in: The Upper Mesosphere and Lower Thermosphere: A Review of Experiment and Theory, Geophys. Monogr. Ser., 87, edited by: Johnson, R. M., and Killeen, T. L., American Geophysical Union, Washington, DC, 23-36, 1995.
Germany, G. A., Parks, G. K., Brittnacher, M. J., Cumnock, J., Lummerzheim, D., Spann, J. F., Chen, L., Richards, P. G., and Rich, F. J.: Remote determination of auroral energy characteristic during substorm activity, Geophys. Res. Lett., 24, 995-998, 1997.

Germany, G. A., Spann, J. F., Parks, G. K., Brittnacher, M. J., Elsen, R., Chen, L., Lummerzheim, D., and Rees, M. H.: Auroral observations from POLAR ultraviolet imager (UVI), in: Geospace Mass and Energy Flow: Results From the International SolarTerrestrial Physics Program, Geophys. Monogr. Ser., vol 104, edited by: Horwitz, J., Gallagher, D., and Peterson, W., AGU, Washington, D.C., 149-160, 1998.

Germany, G. A., Spann, J. F., Deverapalli, C., and Hung, C.: The utility of auroral image-based activity metrics, Eos. Trans. AGU, 85(47), Fall Meet. Suppl., Abstract SA51B-0247, 2004.

Hardy, D. A., Gussenhoven, M. S., Raistrick, R., and McNeil, W. J.: Statistical and functional representations of auroral energy flux, number flux and conductivity, J. Geophys. Res., 92, $12275-$ $12294,1987$.

Janhunen, P.: GUMICS-3: A global ionosphere-magnetosphere coupling simulation with high ionospheric resolution, in: Proc. of Environmental Modelling for Space-Based Applications, Sept. 18-20 1996, Eur. Space Agency Spec. Publ., ESA SP-392, 233-239, 1996.

Janhunen, P.: Reconstruction of electron precipitation characteristics from a set of multiwavelength digital all-sky auroral images, J. Geophys. Res., 106, 18 505-18 516, 2001.

Kamide, Y. and Richmond, A. D.: Ionospheric conductivity dependence of electric field and current estimates from ground-based observations, J. Geophys. Res., 87, 8331-8337, 1982.

Knipp, D. J., Emery, B. A., Engebretson, M., Li, X., McAllister, A. H., Mukai, T., Kakubun, S., Reeves, G. D., Evans, D., Obara, T., Pi, X., Rosenberg, T., Weatherwax, A., McHarg, M. G., Chun, F., Mosely, K., Codrescu, M., Lanzerotti, L., Rich, F. J., Sharber, J., and Wilkinson, P.: An overview of the early November 1993 geomagnetic storm, J. Geophys. Res., 103, 26 197-26 220, 1998.

Liou, K., Newell, P. T., and Meng, C.-I.: Seasonal effects on auroral particle acceleration and precipitation, J. Geophys. Res., 106, 5531-5542, 2001.

Liou, K., Carbary, J. F., Newell, P. T., Meng, C.-I., and Rasmussen, O.: Correlation of auroral power with the polar cap index, J. Geophys. Res., 108(A3), doi:10.1029/2002JA009556, 2003.

Lu, G., Baker, D. N., McPherron, R. L., Farrugia, C. J, Lummerzheim, D., Ruohoniemi, J. M., Rich, F. J., Evans, D. S., Lepping, R. P., Brittnacher, M., Li, X., Greenwald, R., Sofko, G., Villain, J., Lester, M., Thayer, J., Moretto, T., Milling, D., Troshichev, O., Zaitzev, A., Odintzov, V., Makarov, G., and Hayashi, K.: Global energy deposition during the January 1997 magnetic cloud event, J. Geophys. Res., 103, 11 685-11694, 1998.

Lummerzheim, D., Rees, M. H., Carve, J. D., and Frank, L. A.: Ionospheric conductances derived for DE-1 auroral images, J. Atmos. Terr. Phys., 53, 281-292, 1991.

Lummerzheim, D., Brittnacher, M., Evans, D., Germany, G. A., Parks, G. K., Rees, M. H., and Spann, J. F.: High time resolution study of the hemispheric power carried by energetic electrons into the ionosphere during the May 19/20, 1996 auroral activity, Geophys. Res. Lett., 24, 987-990, 1997. 
Newell, P. T., Lyons, K. M., and Meng, C.-I.: A large survey of electron acceleration events, J. Geophys. Res., 101, 2599-2614, 1996.

Østgaard, N., Vondrak, R. R., Gjerloev, J. W., and Germany, G. A.: A relation between the energy deposition by electron precipitation and geomagnetic indices during substorms, J. Geophys. Res., 107(A9), doi:10.1029/2001JA002003, 2002a.

Østgaard, N., Germany, G. A., Stadsnes, J., and Vondrak, R. R.: Energy analysis of substorms based on remote sensing techniques, solarwind measurements and geomagnetic indices. J Geophys. Res., 107(A9), doi:10.1029/2001JA002002, $2002 b$.

Palmroth, M., Janhunen, P., Pulkkinen, T. I., and Peterson, W. K.: Cusp and magnetopause locations in global MHD simulation, J. Geophys. Res., 106, 29 435-29 450, 2001.

Palmroth, M., Janhunen, P., Pulkkinen, T. I., and Koskinen, H. E. J.: Ionospheric energy input as a function of solar wind parameters: global MHD simulation results, Ann. Geophys., 22, 549-566, 2004a.

Palmroth, M., Pulkkinen, T. I., Janhunen, P., and Koskinen, H. E. J.: Ionospheric power consumption in global MHD simulation predicted from solar wind measurements, IEEE Trans. on Plasma Sci., 32, 1511-1518, 2004b.
Palmroth, M., Janhunen, P., Pulkkinen, T. I., Aksnes, A., Lu, G., Østgaard, N., Watermann, J., Reeves, G. D., and Germany, G. A.: Assessment of ionospheric Joule heating by GUMICS-4 MHD simulation, AMIE, and satellite-based statistics: Towards a synthesis, Ann. Geophys., 23, 2051-2068, 2005.

Pulkkinen, T. I., Ganushkina, N. Yu., Tanskanen, E. I., Lu, G., Baker, D. N., Turner, N. E., Fritz, T. A., Fennel, J. F., and Roeder, J.: Energy dissipation during a geomagnetic storm: May 1998, Adv. Space Res., 30, 2231-2240, 2002.

Slinker, S. P., Fedder, J. A., Emery, B. A., Baker, K. B., Lummerzheim, D., Lyon, J. G., and Rich, F. J.: Comparison of global MHD simulations with AMIE simulations for the events of May 19-20, 1996, J. Geophys. Res., 104, 28 379-28 395, 1999.

Tanskanen, E. I., Pulkkinen, T. I., Koskinen, H. E. J., and Slavin, J. A.: Substorm energy budget near solar minimum and maximum: 1997 and 1999 compared, J. Geophys. Res., 107, doi:10.1029/2001JA900153, 2002.

Torr, M. R., Torr, D. G., Zukic, M., Johnson, R. B., Ajello, J., Banks, P., Clark, K., Cole, K., Keffer, C., Parks, G., Tsurutani, B., and Spann, J.: A far ultraviolet imager for the international solar-terrestrial physics mission, Space Sci. Rev., 71, 329-383, 1995.

Vasyliunas, V. M., Kan, J. R., Siscoe, G. L., and Akasofu, S.I.: Scaling relations governing magnetospheric energy transfer, Planet. Space Sci., 30(4), 359-365, 1982. 